\title{
Recent developments in microstructured fiber Bragg grating refractive index sensors
}

\author{
Bin-bin Luo, ${ }^{\text {a,b, }}{ }^{*}$ Xiao-jun Zhou, ${ }^{a}$ Ming-fu Zhao, ${ }^{\text {b, }}$ Nian-bing Zhong, ${ }^{b}$ \\ Sao-fei Wang ${ }^{\mathrm{b}}$ \\ ${ }^{a}$ University of Electronic Science and Technology of China, Department of Photoelectric \\ Information, SC, Chengdu, D.C. 610054, China \\ ${ }^{\mathrm{b}}$ Chongqing University of Technology, Department of Electronic Engineering, Chongqing, \\ D.C. 400050, China \\ *Corresponding authors: luobinbin@cqut.edu.cn, zmf@,cqut.edu.cn
}

\begin{abstract}
Refractive index measurement by optical fiber sensors has proved to be effective in the research of biochemical and biomedical applications. The theoretical principles and technology underlying several microstructured fiber Bragg grating refractive index sensors developed over the past decade are classified and briefly summarized, and their future developments are considered. ๑ 2010 Society of Photo-Optical Instrumentation Engineers. [DOI: 10.1117/6.0000002]
\end{abstract}

Keywords: surrounding refractive indexes; fiber Bragg gratings; etching processes; core modes; cladding modes; single-mode fibers; multimode fibers.

Paper SR090106 received Apr. 6, 2009; revised manuscript received Sep. 8, 2009; accepted for publication Sep. 9, 2009; published online Jan. 14, 2010.

\section{Introduction}

Optical fiber biosensors have been widely used in biochemical and biomedical applications due to such characteristics as immunity to electromagnetic interference and aggressive environments, small size, high sensitivity, and fast response [1-3]. Refractive index sensing has played an especially important role because a number of physical parameters, chemical parameters, and substances can be detected through measurements of refractive index. For example, the refractive index of solutions and gases usually increases linearly with concentration and density, respectively [4]; the $\mathrm{pH}$ values of certain solutions can be measured by their refractive index [5]; the refractive index of biofilm increases from 1.330 to 1.335 as the biofilm thickness increases from 0 to $2 \mathrm{~mm} \mathrm{[6];} \mathrm{antigen-antibody} \mathrm{tests} \mathrm{can} \mathrm{be} \mathrm{based} \mathrm{on} \mathrm{refractive} \mathrm{index} \mathrm{variations} \mathrm{of} \mathrm{bio-}$ logical tissue [7]; and DNA can be detected by measuring the changes of refractive index on the surface of the fiber [8]. Considerable effort has thus been devoted to the invention of diversified fiber sensors for refractive index sensing, including those based on evanescent fields [9], long-period fiber gratings (LPFG) [10], microstructured fiber Bragg gratings (FBG) [11], and so on.

Of the various fiber refractive index sensors, those based on a microstructured FBG exhibit the advantages of wavelength demodulation, absolute measurement, and wavelength multiplexing [12,13]. Additionally, a normal FBG, usually with a length ranging from 1 to $20 \mathrm{~mm}$ and a diameter of $125 \mu \mathrm{m}$, qualifies as one of the smallest sensors in the world, making it especially suitable for refractive index sensing in the biochemical and biomedical fields [14]. Since the invention of the FBG in 1978, FBG sensors have been used mainly in temperature and strain sensing, operating on the principle that the effective refractive index of the fundamental core mode and pitch length change with the outer temperature or strain and thereby cause the Bragg wavelengths to shift $[15,16]$. In the past decade, some kinds of microstructured FBG sensors made by chemical etching processes have been developed for refractive index sensing, most

$1946-3251 / 2010 / \$ 25.00$ @ 2010 SPIE 
of them based on the principle that the resonance wavelengths of the fundamental core mode and/or the cladding modes in single-mode fiber (SMF), or of the high-order core modes in fewmode fiber and multimode fiber (MMF), change with the variation of surrounding refractive index (SRI) [17-19]. The SRI can thus be obtained through measuring the wavelength shift of resonance modes in these microstructured FBGs.

In this review, various microstructured FBG refractive index sensors developed over the past ten years are presented and classified, their underlying theoretical principles are analyzed in detail, and their SRI sensing characteristics are compared. Future developments in this area are then considered.

\section{Basic Theory of Microstructured FBG Refractive Index Sensors}

As is well known, the FBG resonance condition can be expressed as [20]:

$$
\begin{gathered}
\lambda_{B}^{\mathrm{co}}=2 n_{\mathrm{eff}}^{\mathrm{co}} \Lambda_{\mathrm{FBG}}, \\
\lambda_{B}^{v}=\left(n_{\mathrm{eff}}^{\mathrm{co}}+n_{\mathrm{eff}}^{\mathrm{cl}, v}\right) \Lambda_{\mathrm{FBG}},
\end{gathered}
$$

where $\Lambda_{\mathrm{FBG}}$ is the FBG period, $\lambda_{B}^{\mathrm{co}}$ and $\lambda_{B}^{v}$ are the resonance wavelength of the fundamental core mode and the $v^{\text {th }}$-order cladding mode respectively, and $n_{\text {eff }}^{\mathrm{co}}$ and $n_{\mathrm{eff}}^{\mathrm{cl} v}$ are the effective refractive index of the fundamental core mode and the $v^{\text {th }}$-order cladding mode, respectively. According to the mode analysis of a double-cladding fiber geometry model, both $n_{\mathrm{eff}}^{\mathrm{co}}$ and $n_{\mathrm{eff}}^{\mathrm{cl}, v}$ vary with the SRI. Theoretically, we could thus measure the shift of either $\lambda_{\mathrm{B}}^{\mathrm{co}}$ or $\lambda_{B}^{v}$ to detect the variations of the SRI. Practically speaking, a normal FBG is intrinsically insensitive to the SRI because its fundamental core mode is well restricted to the fiber core, and its cladding modes are so well protected by the cladding that they are independent of the SRI. Therefore, a normal FBG is not suited to be a refractive index sensor. Nevertheless, if the cladding of a FBG is etched to the extent that the evanescent field of the fundamental core mode, high-order core modes, or cladding modes can easily interact with the SRI, then a microstructured FBG refractive index sensor is obtained. The many kinds of microstructured FBG refractive index sensors that have been developed in recent years are classified in this review as four types according to their structure and refractive sensing principle. The first type is the cladding-etched SMF FBG; the second is the core-etched SMF FBG; the third is the cladding-core-etched MMF FBG; and the fourth is the nonuniform-etched FBG. The structure of the first three kinds of FBGs is symmetrical, whereas the structure of the last kind of FBG is not. These four kinds of FBGs are inspected in detail in the following sections.

\section{Cladding-Etched SMF FBG}

The structure of a thinned single-mode fiber with its cladding etched by chemical etching in the FBG region is shown in Fig. 1. Unlike the case for temperature and strain measurement, in the case of SRI sensing research, the effective refractive index $n_{\text {eff }}$ is only affected by SRI changes, while the surrounding temperature is assumed to be unchanged. The effective refractive index

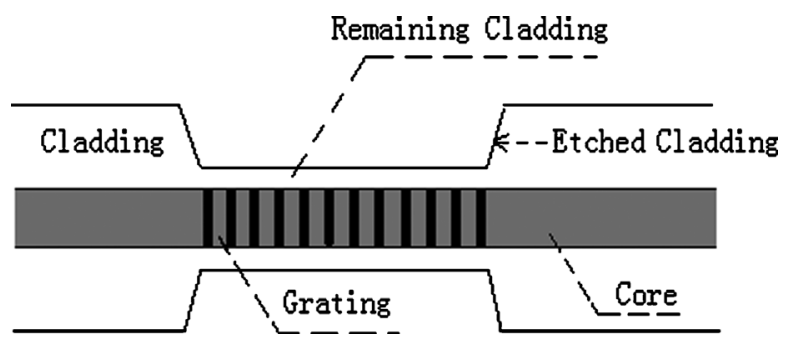

Fig. 1 Profile of cladding-etched SMF FBG. 
Luo et al.: Recent developments in microstructured fiber Bragg grating refractive index sensors

Table 1 Sensor sensitivity in terms of $\left(\Delta \lambda_{B} / \lambda_{B}\right) /\left(\Delta n_{\text {out }} / n_{\text {out }}\right)$ for different cladding diameters and for different surrounding refractive index. Reprinted with permission from A. Cusano, "Thinned fiber Bragg gratings as high sensitivity refractive index sensor," IEEE Photon. Technol. Lett. 16(4), 1149-1151 (2004), ( 2004 IEEE.

\begin{tabular}{llllll}
\hline \hline & Full Etching & $\boldsymbol{D}_{\text {clad }}=\mathbf{1 0} \boldsymbol{\mu \mathbf { m }}$ & $\boldsymbol{D}_{\text {clad }}=\mathbf{1 2} \boldsymbol{\mu \mathbf { m }}$ & $\boldsymbol{D}_{\text {clad }}=\mathbf{1 6} \boldsymbol{\mu \mathbf { m }}$ & $\boldsymbol{D}_{\text {clad }}=\mathbf{2 0} \boldsymbol{\mu \mathbf { m }}$ \\
\hline$n_{\text {out }}=1.35$ & 4.45 & 2.01 & $7.84 \times 10^{-1}$ & $1.69 \times 10^{-1}$ & $3.88 \times 10^{-2}$ \\
$n_{\text {out }}=1.40$ & $1.04 \times 10^{1}$ & 4.79 & 1.99 & $4.03 \times 10^{-1}$ & $9.28 \times 10^{-2}$ \\
$n_{\text {out }}=1.45$ & $9.05 \times 10^{1}$ & $4.34 \times 10^{1}$ & $1.92 \times 10^{1}$ & 4.23 & 1.01 \\
\hline \hline
\end{tabular}

of both the core modes and the cladding modes is sensitive to the SRI. However, for many practical applications, temperature is an important factor, and thus great effort has been made to eliminate the impact of temperature on FBG refractive index sensors.

\subsection{Characteristics of the Fundamental Core Mode in the Cladding-Etched SMF FBG}

The behavior of the effective refractive index of the fundamental core mode as a function of the SRI in a cladding-etched single-mode FBG has been investigated by Iadicicco and Cusano [21]. They selected a SMF-28 fiber with a core diameter of $8.3 \mu \mathrm{m}$, an original cladding diameter of $125 \mu \mathrm{m}$, a core refractive index of 1.460 , a refractive index difference of $0.36 \%$ for numerical analysis and experiment, and an effective refractive index $n_{\text {eff }}$ of the fundamental core mode of 1.4588 at an operating wavelength of $1550 \mathrm{~nm}$. Based on the double-cladding fiber model theory, the numerical analysis showed that the effective refractive index of the fundamental core mode was not sensitive to the SRI until the cladding was etched to a diameter smaller than $20 \mu \mathrm{m}$; as the cladding was etched further, the fundamental core mode resonance wavelength $\lambda_{B}$ decreased for the same SRI, and the relative sensitivity of the sensors, defined as $\left(\Delta \lambda_{B} / \lambda_{B}\right) /\left(\Delta n_{\text {out }} / n_{\text {out }}\right)$, increased, where $n_{\text {out }}$ is the SRI and $\Delta \lambda_{B}$ and $\Delta n_{\text {out }}$ are the variation of $\lambda_{B}$ and $n_{\text {out }}$, respectively. In addition, with the same cladding diameter, $n_{\text {eff }}$ showed a nonlinear relationship with the SRI $n_{\text {out }}$ and increased more and more rapidly with $n_{\text {out }}$ growing from 1.33 to that of the cladding, causing the resonance wavelength $\lambda_{B}$ to move to a longer wavelength in accord with Eq. (1). A higher relative sensitivity $\left(\Delta \lambda_{B} / \lambda_{B}\right) /\left(\Delta n_{\text {out }} / n_{\text {out }}\right)$ was observed as well, since the fundamental core mode was less confined in the core region, leading to an increased interaction with the surrounding medium. The theoretical calculation for SRI sensitivity is shown in Table 1 [21], and the experimental curve of wavelength shift versus SRI in a SMF FBG with core diameter of $8.5 \mu \mathrm{m}$ is shown in Fig. 2 [21].

A comparison of SRI sensitivity of the fundamental core mode for cladding-etched SMF FBGs with different core diameters has been completed by Xia et al. [22]. Their numerical analysis showed that the SRI sensitivity of the cladding-etched FGB with a thinner core diameter was larger than that of a thicker one. Thus, in order to increase the SRI sensitivity, a small-corediameter FBG should be chosen for the cladding etching process.

\subsection{Characteristics of the Cladding Modes in the Cladding-Etched SMF FBG}

Characteristics of the cladding modes in the cladding-etched SMF FBG have been researched by Chen $[23,24]$, who utilized the double-cladding fiber model theory. Parameters for the numerical calculation were as follows: fiber core diameter $2 a_{1}=6.4 \mu \mathrm{m}$, fiber cladding diameter $2 a_{2}=124.7 \mu \mathrm{m}$, refractive index difference $\Delta=0.45 \%$, SRI $n_{3}=1.00$, grating length $L=$ $8 \mathrm{~mm}$, fringe visibility of index change $m=0.6$, average index change over a grating period $\overline{\delta n}=4.153 \times 10^{-4}$, and grating period $\Lambda_{\mathrm{FBG}}=526.648 \mathrm{~nm}$. The numerical analysis in Ref. 23 showed that the resonance cladding modes of an unetched SMF FBG are too weak and too 


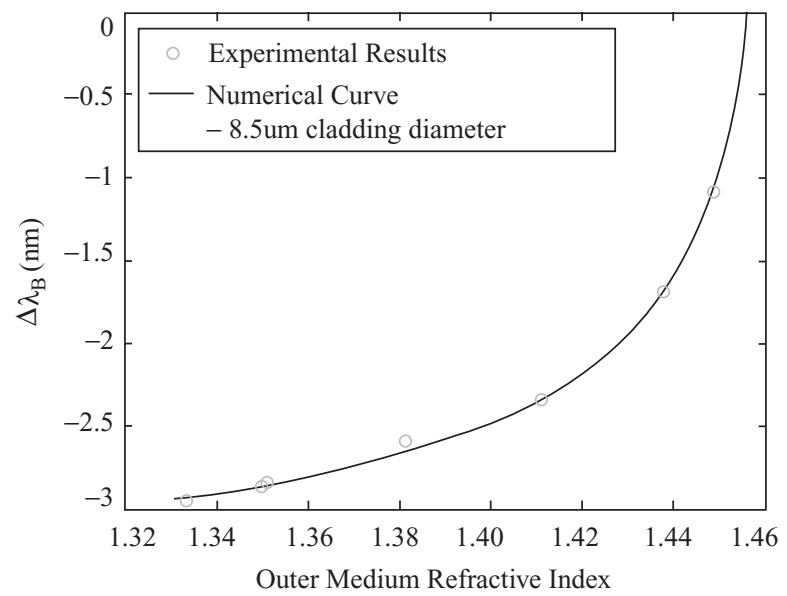

Fig. 2 Wavelength shift of the reflected signal of a cladding-etched SMF FBG (dotted line) and the numerical curve (solid line) with a cladding diameter of $8.5 \mu \mathrm{m}$ versus external medium. Reprinted with permission from A. Cusano, "Thinned fiber Bragg gratings as high sensitivity refractive index sensor," IEEE Photon. Technol. Lett. 16(4), 1149-1151 (2004), ๑ 2004 IEEE.

dense to be discriminated from one another in real applications. However, with a decrease of the cladding diameter to $66.4 \mu \mathrm{m}$, the number of resonance cladding modes began to decrease significantly, the coupling strength grew, the distance between two resonance cladding modes increased, and the bandwidth of the resonance wavelengths became narrower, making it possible to observe the cladding-mode resonance wavelength with a spectral instrument. As the SRI increases under the condition of an unchanged cladding diameter, the effective refractive index of the $v^{\text {th }}$-order cladding mode $n_{\mathrm{eff}}^{\mathrm{cl}, v}$ increases more and more rapidly, causing the cladding resonance wavelength $\lambda_{B}^{v}$ to shift to a longer wavelength, in accord with Eq. (2), and the sensitivity of the $v^{\text {th }}$-order cladding mode defined by $\left(\Delta \lambda_{B}^{v}\right) /\left(\Delta n_{\text {out }}\right)$ to increase as the SRI approaches the index of the cladding (the typical value of a fiber cladding index is about 1.44). In addition, the higher the order of a cladding mode used for SRI measurement, the more sensitive the device is to the SRI, as shown in Fig. 3 [23], since the evanescent field of a high-order cladding mode interacts with the outer medium more easily than does a lower order mode.

As the cladding of a FBG is etched deeper and deeper, the mechanical strength of the fiber decreases. Thus, in order to obtain higher refractive index sensitivity and at the same time maintain mechanical strength, it is better to operate a cladding-etched FBG in the high-order cladding modes for SRI measurement instead of the fundamental core mode, since the etching depth is not so deep. Experiments carried out by Yun [25] showed the highest refractive index sensitivity of $172 \mathrm{~nm}$ per refractive index unit (RIU) by using the fourth-order cladding mode in a cladding-etched FBG, larger than the $71.2 \mathrm{~nm} / \mathrm{RIU}$ [26] achieved by the fundamental core-core mode coupling.

\subsection{Characteristics of the Few-Mode Application in the Cladding-Etched SMF FBG}

A SMF FBG can be referred to as a few-mode-fiber FBG if the following conditions are met: more than one core mode can transmit in a fiber core if the operating wavelength is small enough to insure that the normalized frequency $V$ is larger than 2.045, but at the same time, fewer than ten core modes transmit in the fiber core.

Numerical analysis and experiments involving a cladding-etched few-mode-fiber FBG made with a normal G.652 SMF are reported in Ref. 27. Under the condition that the operating wavelength is from 1000 to $1100 \mathrm{~nm}$, the normal G.652 SMF operates in the few-mode application 


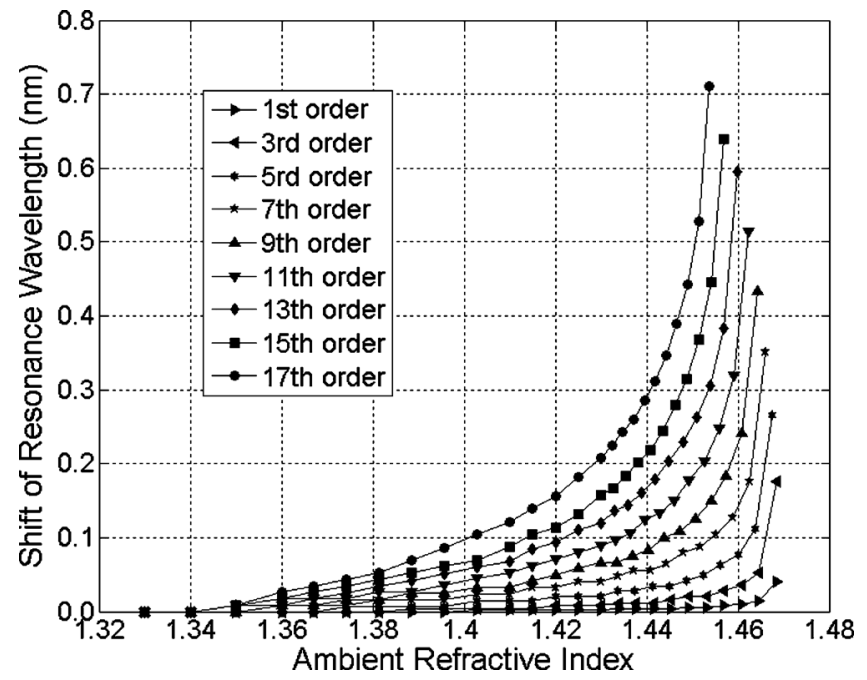

Fig. 3 Shifts of $17^{\text {th }}, 15^{\text {th }}, 13^{\text {th }}, 11^{\text {th }}, 9^{\text {th }}, 7^{\text {th }}$, and $5^{\text {th }} \mathrm{SRI}$ in a $3^{\text {rd }}$ and $1^{\text {st }}$ order cladding mode resonance with SRI under a fiber diameter of $66.4 \mu \mathrm{m}$. Reprinted with permission from Y. Cui, "Theoretical and experimental study on etched fiber Bragg grating cladding mode resonances for ambient refractive index sensing," J. Opt. Soc. Am. B 24(3), 439-445 (2007), ( 2007 OSA.

area. In this case, the fundamental core-core mode coupling $L P_{\mathrm{co} \rightarrow \mathrm{co}}^{01 \rightarrow 01}$ and the coupling of the fundamental core mode to its first high-order mode $L P_{\mathrm{co} \rightarrow 00}^{01 \rightarrow 02}$ existed in the FBG. When the cladding was etched down to a remaining thickness smaller than $3.5 \mu \mathrm{m}$, both $L P_{\mathrm{co} \rightarrow \mathrm{co}}^{01 \rightarrow 01}$ and $L P_{\mathrm{co} \rightarrow \mathrm{co}}^{01 \rightarrow 02}$ began to shift to a shorter wavelength. As the SRI increased, both the Bragg wavelength of $L P_{\mathrm{co} \rightarrow \mathrm{co}}^{01 \rightarrow 01}$ and $L P_{\mathrm{co} \rightarrow \mathrm{co}}^{01 \rightarrow 02}$ increased more and more rapidly. Furthermore, the SRI sensitivity of $L P_{\mathrm{co} \rightarrow \mathrm{co}}^{01 \rightarrow 02}$ was always higher than that of $L P_{\mathrm{co} \rightarrow \mathrm{co}}^{01 \rightarrow 01}$, since the evanescent wave of $L P_{02}$ interacts with the SRI more easily than does the fundamental core mode $L P_{01}$. In addition, the temperature dependency of $L P_{\mathrm{co} \rightarrow \mathrm{co}}^{01 \rightarrow 01}$ and $L P_{\mathrm{co} \rightarrow \mathrm{co}}^{01 \rightarrow 02}$ are the same from $10^{\circ} \mathrm{C}$ to $52{ }^{\circ} \mathrm{C}$, making it possible to carry out temperature compensation in the SRI measurement by one cladding-etched few-mode-fiber FBG.

\section{Core-Etched SMF FBG}

Unlike that of the cladding-etched SMF FBG, the numerical analysis of a core-etched FBG is based on the single-cladding geometrical model, in which the FBG cladding is substituted by the surrounding media for analysis. In this case, the fundamental core mode is considered, and if the few-mode conditions are met, some high-order core modes are also considered.

By solving the modal equation of the single-cladding geometrical model, the result of numerical calculation between the normalized transmission constant $b$ versus normalized frequency $V$ in a fiber is plotted with the blue curves in Fig. 4. Meanwhile, the functional dependence of $b$ upon $V$ in a core-etched FBG with a core diameter $a$ is given by [28]

$$
b=\frac{(\beta / k)^{2}-n_{2}^{2}}{n_{1}^{2}-n_{2}^{2}}=\frac{\left(\lambda_{B} / 2 \Lambda\right)^{2}-n_{2}^{2}}{n_{1}^{2}-n_{2}^{2}}=\frac{(\pi a / V \Lambda)^{2}\left(n_{1}^{2}-n_{2}^{2}\right)-n_{2}^{2}}{n_{1}^{2}-n_{2}^{2}},
$$

where $n_{1}$ is the index of the fiber core, $n_{2}$ is the index of the cladding (surrounding medium), $\lambda_{B}$ is the Bragg wavelength of one of the resonance core modes, $\beta$ is the correspondent propagation constant, $k$ is the wave number in vacuum, and $\Lambda$ is the FBG period. The curves of Eq. (3) in the wavelength windows between 1.5 and $1.6 \mu \mathrm{m}$ for different core diameters are plotted by the straight lines in Fig. 4 [28]. It is clear that the intersection of the blue curves and straight lines 


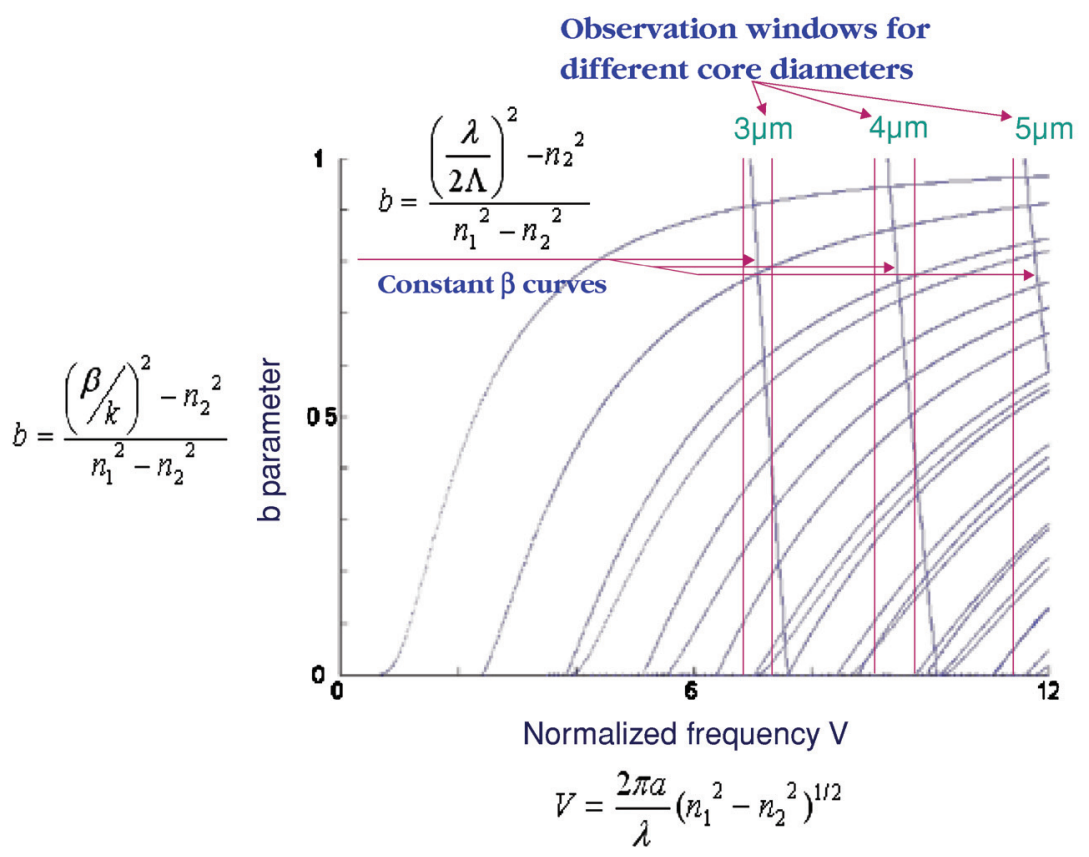

Fig. 4 The curve of normalized propagation $b$ versus $V$ and the curves of constant grating. Reprinted with permission from A. N. Chryssis, "High sensitivity evanescent field fiber Bragg grating sensor," Photon. Technol. Lett. 17(6), 1253-1255 (2005), ๑ 2005 IEEE.

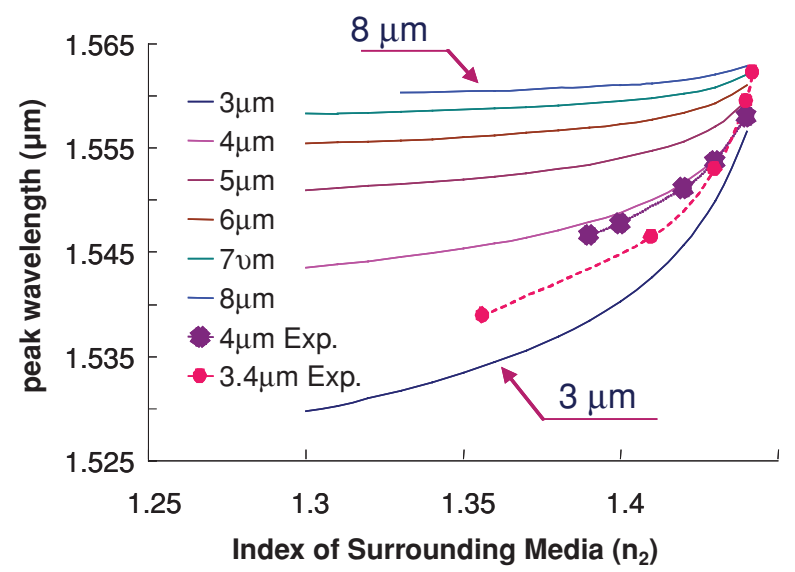

Fig. 5 Change in Bragg wavelength with changing surrounding index for different diameters of fiber. Reprinted with permission from A. N. Chryssis, "High sensitivity evanescent field fiber Bragg grating sensor," Photon. Technol. Lett. 17(6), 1253-1255 (2005), ๑ 2005 IEEE.

can give the Bragg wavelength $\lambda_{B}$ of the correspondent resonance core modes; on the other hand, if $\lambda_{B}$ is detected, $n_{2}$ (SRI) will be obtained from Eq. (3).

Some of the simulation and experimental results are summarized here. First, as the fiber core is etched, the Bragg wavelength $\lambda_{B}$ decreases for the same SRI. Second, the Bragg wavelength $\lambda_{B}$ changes faster in a thinner-core-diameter FBG than it does in a thicker FBG, other conditions being the same, with a corresponding increase in sensitivity for the sensor. Also, the SRI sensitivity is higher when the SRI is close to the index of the core (the typical value of a fiber core index is about 1.460), as shown in Fig. 5 [28].

Experiment research on refractive-index sensitivity of the fundamental core mode in a coreetched FBG that was used to detect DNA is reported in Ref. 29. The fiber core was etched 
to $3.4 \mu \mathrm{m}$, and the SRI increased until it nearly equaled the index of the core. The highest sensitivity $\left(\Delta \lambda_{\mathrm{B}}\right) /\left(\Delta n_{\text {out }}\right)$ achieved was $1394 \mathrm{~nm} / \mathrm{RIU}$, a value higher than that achieved with the cladding-etched FBG mentioned above.

If the SRI is small enough to insure that the normalized frequency $V$ is larger than 2.045, then some high-order core modes will appear in a core-etched SMF FBG, leading to a few-mode application. Numerical analysis in Ref. 30 shows that the SRI sensitivity increases with the order of core modes, other conditions being the same, the sensitivity of the third-order core mode increasing by at least a factor of 6 as compared to that of the fundamental core mode for a FBG etched down to a core diameter of $5 \mu \mathrm{m}$. However, the changes in the resonance wavelength of all the existing core modes are the same in the case when only the physical properties of the fiber, such as the core refractive index and the grating period, are altered. This is not the case when the SRI is changed. Therefore, on the one hand, in order to achieve the maximum SRI sensitivity, the wavelength shift of the highest order core mode of a core-etched FBG should be measured. On the other hand, if the observed shift for the higher order core modes is larger than that of the fundamental core mode, then the wavelength change is due to the change of SRI. This principle can be used for the simultaneous measurement of temperature and SRI by one core-etched FBG sensor operating in the few-mode application area.

\section{Cladding-Core-Etched Multimode Fiber FBG}

Since the core diameter of a multimode fiber is approximately $50 \mu \mathrm{m}$, its cladding can be removed by chemical etching more easily than that of a single-mode fiber. Multimode fibers where both the cladding and the core are etched are referred to as cladding-core-etched MMF FBG in this review. More than one core mode can propagate and satisfy the phase matching conditions in MMF FBGs, and the mode coupling in a MMF FBG is thus more complicated than in SMF FBGs. The mode coupling theory and temperature dependence characteristics in a MMF FBG with a core diameter of $50 \mu \mathrm{m}$ and numerical aperture (NA) of 0.2 have been researched in detail in Ref. 31, the results of which showed that, for a few-mode application, the Bragg grating in MMF at around $1.55 \mu \mathrm{m}$ has 4 reflection wavelengths. By way of contrast, for a multimode application, the same FBG showed 19 reflection wavelengths, 10 of them attributed to a same core mode coupling and the rest attributed to the reflection to the neighboring core modes. In addition, all the reflection peaks had almost the same temperature dependence of $11.4 \mathrm{pm} /{ }^{\circ} \mathrm{C}[31]$, suggesting the possibility of the simultaneous measurement of temperature and SRI through one cladding-core-etched MMF FBG.

The experiments for a cladding-core-etched MMF FBG with NA $=0.22, D_{\mathrm{co}}=50 \mu \mathrm{m}$, and a tilted angle of $0.7 \mathrm{deg}$ for SRI sensing are reported in Ref. 32. Before the etching process, the fundamental core-core mode $L P_{\mathrm{co} \rightarrow \mathrm{co}}^{01 \rightarrow 01}$ was the most strongly coupled. The core-core mode coupling decreased linearly with increasing mode order. When the MMF FBG cladding was etched beyond $160 \mathrm{~min}$, high-order core modes began to be blue-shifted, whereas the lower order core modes remained the same. When the cladding was etched beyond $250 \mathrm{~min}$, the lower order core modes began to be blue-shifted at increasing rates with increasing etching time and mode order as well. When the MMF FBG core was etched to a $12-\mu \mathrm{m}$ remaining core diameter, there were only two or three modes remaining in the core. These were investigated for SRI sensitivity by measuring a sugar solution with concentrations ranging from $0 \%$ to $60 \%$, corresponding to the SRI ranging from 1.33 to 1.44. Experimental results showed that all existing core modes shifted to a longer wavelength more and more rapidly as the SRI increased, and the wavelength shift of $L P_{\mathrm{co} \rightarrow \mathrm{co}}^{01 \rightarrow 11}$ was approximately two times that of $L P_{\mathrm{co} \rightarrow \mathrm{co}}^{01 \rightarrow 01}$ [32].

\section{Nonuniform-Etched FBG}

In this review, the term nonuniform-etched FBG refers to a special etching style, such as a D-shaped FBG, a partially etched FBG, and so on. This type of microstructured FBG is made using a single-mode fiber, and thus only the fundamental core mode can be employed for SRI 


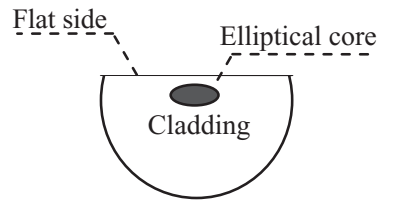

Fig. 6 Cross-section structure of D-shaped FBG with elliptical core.

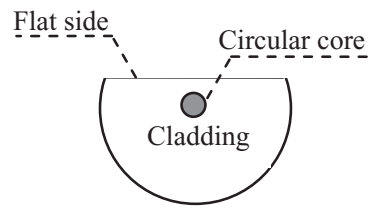

Fig. 7 Cross-section structure of D-shaped FBG with circular core.

measurement. A typical D-shaped FBG is manufactured with an elliptical core with a short axis of $2.5 \mu \mathrm{m}$ and a long axis of $4.5 \mu \mathrm{m}$ [33]. The thickness of the residual cladding layer on the flat side is reduced to just a few microns, as shown in Fig. 6. The Bragg wavelength shifts dramatically toward the shorter wavelengths, and the transmission loss strength (Bragg reflection) decreases as the etching process continues, due to the combined effects of the reduction of the effective mode index and the degradation of the light confinement. Research reported in Ref. 33 also compares the SRI sensitivity of the elliptical core D-shaped FBGs with different etching depths and reveals that the SRI sensitivity, as for the other three types of microstructure FBGs noted, increases with increasing etching depth and increases more and more rapidly as the SRI changes from 1.33 to that of fiber cladding, showing a nonlinear relationship. However, if a D-shaped FBG sensor is made from a normal FBG with a circular core, as shown in Fig. 7, a distinguishing and interesting phenomenon is observed. An experiment reported in Ref. 34 shows that both the Bragg wavelength shift and the variation of Bragg reflection intensity exhibits a linear relation with the concentration of surrounding sugar solutions varying from 0.00 to $4.62 \mathrm{~mol} / \mathrm{L}$, corresponding to the SRI varying from 1.33 to about 1.38 .

The structure of a partially etched FBG is illustrated in Fig. 8. Numerical and experimental results reported in Refs. 35 and 36 reveal that the single-peak Bragg wavelength of the fundamental core mode is separated to double-peak wavelengths with a dip between them, due to the formation of a narrow band gap inside the stop-band spectrum of the device, which results from the microstructure perturbation in the partially etched FBG, as shown in Fig. 9. The resonance characteristics of a partially etched FBG are strongly dependent on etching position $L_{1}$ and $L_{2}$, etching depth $\delta_{d}$, etching length $L_{d}$, and SRI $n_{\text {out }}$. The most important characteristic is that the wavelength of the dip increases linearly with SRI increasing from 1.33 to 1.457 , larger than the cladding index, at room temperature. In addition, both the dip wavelength and the first-peak wavelength change linearly with surrounding temperature, but the $\mathrm{D}$ value between them does not change with the surrounding temperature at all. Meanwhile, the intensity of double peaks is almost independent of the temperature and of the SRI varying from 1.33 to that of the cladding.

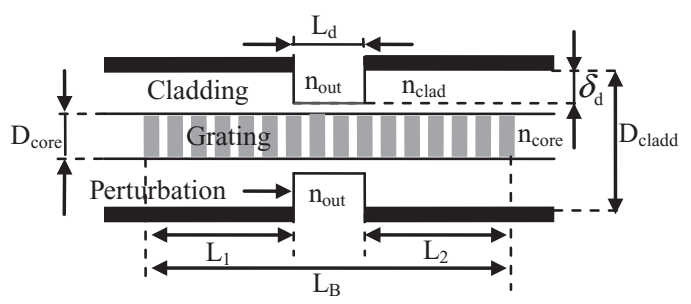

Fig. 8 Profile of partially etched FBG. 


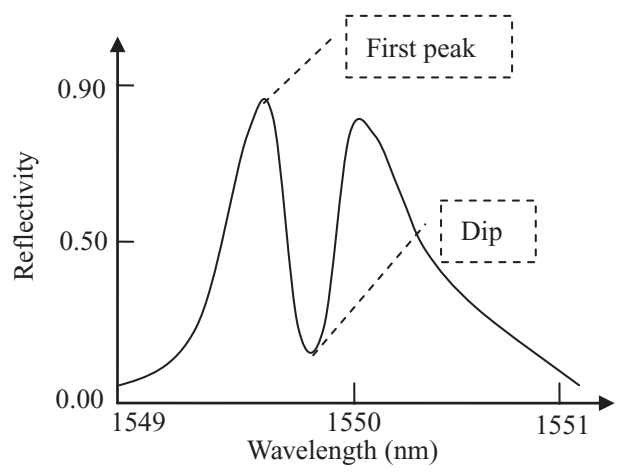

Fig. 9 Double-peak wavelengths in a partially etched FBG.

These conditions mean that the reflectance spectrum profile of the double peaks remains almost unaffected by the disturbance of the surrounding temperature but simply shifts. However, once the SRI increases to be larger than the cladding index, the intensity of double peaks decreases dramatically [36].

\section{Conclusions and Future Developments}

In this review, the microstructured FBG refractive index sensors invented in the last ten years are classified as cladding-etched SMF FBGs, core-etched SMF FBGs, cladding-core-etched MMF FBGs, and nonuniform-etched FBGs. Some comparisons and common characteristics are summarized here.

In the case of the cladding-etched SMF FBG, the fundamental core mode, the existing highorder core modes, and the existing cladding modes may be employed for the SRI measurement, the cladding modes revealing a higher SRI sensitivity than do the former two in the same microstructured FBG. The core-etched SMF FBG reveals the highest SRI sensitivity compared with other types of microstructured FBG SRI sensors. In the case of the cladding-core-etched MMF FBG, multiple resonant core modes can be utilized for SRI measurement, and these devices usually maintain a greater mechanical strength after etching than do the single-mode fiber FBG SRI sensors, but less than do the D-shaped FBG sensors, for the chemical etching being carried out on just one side of the D-shaped FBG. For the partially etched FBG, the reflective wavelength shift of the dip formed by the fundamental core mode is available for SRI measurement. For all of these microstructured FBG SRI sensors, the Bragg wavelength of any coupling mode moves toward shorter wavelengths as the etching depth increases, the deeper etching yielding higher SRI sensitivity for any coupling mode. Also, the Bragg wavelength of any coupling core mode and cladding mode in almost all types of the microstructure FBG increases more and more rapidly as the SRI increases from 1.33 to the index of the core or cladding, showing a nonlinear relationship between the wavelength shift and SRI variation, except for the dip wavelength in the partially etched FBG increasing linearly with the SRI growing from 1.33 to 1.457 , larger than the cladding index. In particular, both the reflective wavelength and intensity of the D-shaped FBG with a circular core exhibit a linear relation with the SRI ranging from 1.33 to about 1.38, smaller than the cladding index. A high-order coupling mode exhibits a higher SRI sensitivity than does a low-order coupling mode under the same conditions, and thus the resonant cladding modes always show a higher SRI sensitivity than do the resonant core modes in the same FBG sensor. Finally, all the existing coupling modes in a cladding-etched SMF FBG, in a core-etched SMF FBG, or in a cladding-core-etched MMF FBG have been shown to possess approximately the same temperature dependence but not the same SRI sensitivity.

Apart from the microstructured FBG SRI sensors mentioned above, there are other special fiber grating sensors for SRI sensing as well, such as hybrid structure gratings in D fiber [37], 
3-deg slanted multimode fiber Bragg gratings [38], tilted FBGs for refractometry [39], sampled FBGs for simultaneous temperature and SRI sensing [40], LPFG SRI sensors [41,42], and so on. But most of them are less sensitive to SRI or less compact than are the microstructured FBGs discussed in this paper. However, they usually have not undergone a chemical etching and would thus retain greater mechanical strength.

With the development of new fiber grating demodulation technologies, manufacturing techniques for microstructured FBGs, and increasing demands on SRI sensing for biochemical and biomedical applications, the future developments of microstructured FBG SRI sensors will aim at higher SRI sensitivity, greater mechanical strength, increased temperature independence, and more compact structure.

\section{Acknowledgments}

This work is supported by the National Natural Science Foundation of China under Project No. 50876120 and the State Key Program of National Natural Science of China under Project No. 90510020 .

\section{References}

[1] D. V. Lim, "Detection of microorganisms and toxins with evanescent wave fiber-optic biosensors," Proc. IEEE 91(6), 902-907 (2003).

[2] G. P. Anderson et al., "Multi-analyte interrogation using the fiber optical biosensor," Biosens. Bioelectron. 14(10), 771-777 (2000).

[3] H. Ming, X.-S. Xu, P. Wang, et al., "Polymer fiber and its application," J. China Inst. Metrol. 12(2), 55-64 (2001).

[4] R. C. West, Ed., Handbook of Chemistry and Physics, CRC Press, Boca Raton, Fla. (1981).

[5] B. J.-C. Deboux, E. Lewis, P. J. Scully, and R. Edwards, "A novel technique for optical fiber pH sensing based on methylene blue adsorption," J. Lightwave Technol. 13(7), 1407-1414 (1995).

[6] R. Philip-Chandy, P. J. Scully, P. Eldridge, et al., "An optical fiber sensor for biofilm measurement using intensity modulation and image analysis," IEEE J. Select. Topics Quantum Electron. 6(5), 764-772 (2000).

[7] M. P. DeLisa, Z. Zhang, M. Shiloach, et al., "Evanescent wave long-period fiber Bragg grating as an immobilized antibody biosensor," Anal. Chem. 72(13), 2895-2900 (2000).

[8] X. Liu and W. Tan, "A fiber-optic evanescent wave DNA biosensor based on novel molecular beacons," Anal. Chem. 71(22), 50-54 (1999).

[9] G. P. Anderson, J. P. Golden, and F. S. Ligler, "An evanescent wave biosensor-part I: fluorescent signal acquisition from step-etched fiber optic probes," IEEE Trans. Biomed. Eng. 41(6), 578-584 (1994).

[10] D. W. Kin, Y. Zhang, K. L. Cooper, and A. Wang, "Fiber-optic interferometric immunosensor using long period grating," Electron. Lett. 42(6), 324-325 (2006).

[11] A. Cusano, A. Iadicicco, et al., "Thinned and micro-structured fibre Bragg gratings: towards new all-fibre high-sensitivity chemical sensors," J. Opt. A: Pure Appl. Opt. 12, 7734-7741 (2005).

[12] K. Schroeder, W. Ecke, R. Mueller, et al., "A fibre Bragg grating refractometer," Meas. Sci. Technol. 12, 757-764 (2001).

[13] K. Zhou, X. Chen, L. Zhang, and I. Bennion, "Implementation of optical chemsensors based on HF-etched fibre Bragg grating structures," Meas. Sci. Technol. 17, 1140-1145 (2006).

[14] G. Meltz, W. W. Morey, and J. R. Dunphy, "Fiber Bragg grating chemical sensor," Proc. SPIE 1587, 350-361 (1991).

[15] Y. J. Rao, D. J. Webb, D. A. Jackson, et al., "In-situ temperature monitoring in NMR machines with a prototype in-fiber Bragg grating sensor system," in Proc. 12th Intl. Conf. Optical Fiber Sensors, pp. 646-647, Optical Society of America (1997). 
Luo et al.: Recent developments in microstructured fiber Bragg grating refractive index sensors

[16] S. E. Kanellopoulos, V. A. Handerek, and A. J. Rogers, "Simultaneous strain and temperature sensing with photo generated in-fiber gratings," Opt. Lett. 20, 333-335 (1995).

[17] A. Iadicicco, S. Campopiano, A. Cutolo, et al., "Refractive index sensor based on microstructured fiber Bragg grating," Photon. Technol. Lett. 17, 1250-1252 (2005).

[18] A. Iadicicco, S. Campopiano, and A. Cutolo, "Self temperature referenced refractive index sensor by non-uniform thinned fiber Bragg gratings," Sens. Actuators B 120, 231-237 (2006).

[19] K. Zhou, A. G. Simpson, L. Zhang, and I. Bennion, "Side detection of strong radiation-mode out-coupling from blazed FBGs in single-mode and multimode fibers," Photon. Technol. Lett. 15(7), 936-938 (2003).

[20] T. Erdogan, "Cladding-mode resonances in short and long-period fiber grating filters," J. Opt. Soc. Am. A 14(8), 1760-1773 (1997).

[21] A. Iadicicco and A. Cusano, "Thinned fiber Bragg gratings as high sensitivity refractive index sensor," IEEE Photon. Technol. Lett. 16(4), 1149-1151 (2004).

[22] X. Zhang, Y. Xia, et al., "Analysis of shift in Bragg wavelength of fiber Bragg gratings with finite cladding radius," Acta Photonica Sinaca 32(12), 222-224 (2003).

[23] N. Chen, B. Yun, Y. Wang, and Y. Cui, "Theoretical and experimental study on etched fiber Bragg grating cladding mode resonances for ambient refractive index sensing," J. Opt. Soc. Am. B 24(3), 439-445 (2007).

[24] N. Chen, B. Yun, and Y. Cui, "Cladding mode resonances of etch-eroded fiber Bragg grating for ambient refractive index sensing," Appl. Phys. Lett. 88, 1339-1342 (2006).

[25] B. Yun, N. Chen, and Y. Cui, "Refractive index sensing characteristics of fiber Bragg grating based on cladding mode," Acta Optica Sinica 26(7), 1013-1015 (2005).

[26] W. Liang, Y. Huang, Y. Xu, et al., "Highly sensitive fiber Bragg grating refractive index sensors," Appl. Phys. Lett. 86(15), 151122 (2005).

[27] J. Xu, J. Li, H. Rong, et al., "Analysis and measurement on refractive index sensing of few mode fiber Bragg gratings," Acta Optica Sinica 28(3), 565-568 (2008).

[28] A. N. Chryssis, S. M. Lee, S. B. Lee, et al., "High sensitivity evanescent field fiber Bragg grating sensor," Photon. Technol. Lett. 17(6), 1253-1255 (2005).

[29] M. Dagenais, A. N. Chryssis, et al., "High sensitivity bio-sensor based on an etched core fiber Bragg grating," in OSA Conf. Integrated Photon. Res. Appl. 4, IWD3 (2005).

[30] A. N. Chryssis, S. S. Saini, et al., "Increased sensitivity and parametric discrimination using higher order modes of etched-Core fiber Bragg grating sensors using," Photon. Technol. Lett. 18(1), 178-180 (2006).

[31] T. Mizunami, T. V. Djambova, et al., "Bragg gratings in multimode and few-mode optical fibers," J. Lightwave Technol. 18(2), 230-235 (2000).

[32] K. Zhou, X. Chen, et al., "Optical chemsensors based on etched fiber Bragg gratings in D-shape and multimode fibers," Proc. SPIE 5855, 158-161 (2005).

[33] K. Zhou, X. Chen, L. Zhang, and I. Bennion, "High-sensitivity optical chemsensor based on etched D-fibre Bragg gratings," Electron. Lett. 40, 232-234 (2004).

[34] L. Shen, S.-L. Zheng, and X.-M. Zhang, "The response of side-polished FBG to external refractive index," Acta Photonica Sinica 34(7), 1036-1038 (2005).

[35] A. Iadicicco, A. Cutolo, S. Campopiano, et al., "Advanced fiber optical refract meters based on partially etched fiber Bragg gratings," in Proc. IEEE Sens. 3, 1218-1221 (2004).

[36] X.-F. Huang, Z.-M. Chen, L.-Y. Shao, et al., "Design and characteristics of refractive index sensor based on thinned and microstructure fiber Bragg grating," Appl. Opt. 47(4), 504-511 (2008).

[37] X. Chen, K. Zhou, et al., "Simultaneous measurement of temperature and external refractive index by use of a hybrid grating in D fiber with enhanced sensitivity by HF etching," Appl. Opt. 44(2), 178-182 (2005).

[38] C.-L. Zhao, X. Yang, M. S. Demokan, et al., "Simultaneous temperature and refractive index measurements using a $3^{\circ}$ slanted multimode fiber Bragg grating," J. Lightwave Technol. 24(2), 879-883 (2006). 
[39] G. Laffont and P. Ferdinand, "Tilted short-period fiber-Bragg-grating induced coupling to cladding modes for accurate refractometry," Meas. Sci. Technol. 12, 765-770 (2001).

[40] X. W. Shu, B. A. L. Gwandu, and Y. Liu, "Sampled fiber Bragg grating for simultaneous refractive-index and temperature measurement," Opt. Lett. 26, 774-776 (2001).

[41] H. J. Patrick, A. D. Kersey, et al., "Analysis of the response of long period fiber gratings to external index of refraction," J. Lightwave Technol. 16(9), 1606-1612 (1998).

[42] K. S. Chiang, Y. Liu, et al., "Analysis of etched long-period fibre grating and its response to external refractive index," Electron. Lett. 36(11), 966-967 (2000).

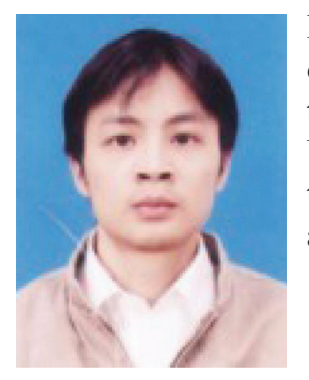

Bin-bin Luo is currently a doctor of optical engineering at the University of Electronic Science and Technology of China, and serves as a lecturer in the Department of Electrical Engineering at the University of Chongqing, University of Technology. His research interests include fiber sensors and techniques, especially the FBG and LPFG refractive index sensing theory and applications in biomedical and biochemical fields.

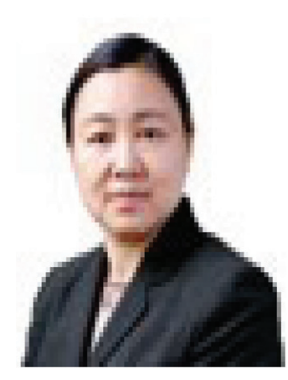

Xiao-jun Zhou is currently a professor at the University of Electronic Science and Technology of China. Her research interests include optical fiber communication and sensors and FBG and LPFG theory and application.

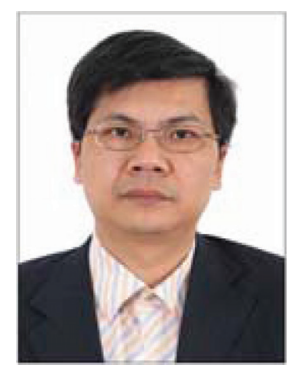

Ming-fu Zhao works as a professor and master tutor in the Department of Electrical Engineering at the University of Chongqing, University of Technology, and part-time tutor of doctoral students at the University of Electronic Science and Technology of China. His research interests include biochemical information extraction and smart optical fiber sensors.

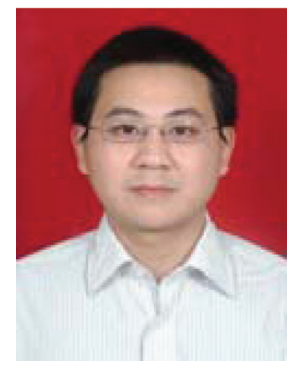

Nian-bing Zhong is studying at the University of Chongqing, University of Technology. He is pursuing a master's degree in biochemical information. 